\title{
ON THE EXISTENCE OF STATIONARY SOLUTIONS FOR SOME NONLINEAR HEAT EQUATIONS
}

\author{
Vitaly Volpert and Vitali Vougalter \\ University Lyon 1, Institute Camille Jordan, UMR 5208 CNRS \\ Villeurbanne, 69622, France; volpert@math.univ-lyon1.fr \\ University of Toronto, Department of Mathematics \\ Toronto, Ontario, M5S 2E4, Canada; vitali@math.toronto.edu
}

\begin{abstract}
We establish the existence in $H^{3}\left(\mathbf{R}^{5}\right)$ of stationary solutions of certain nonlinear heat equations using the Fixed Point Technique. The equation for the perturbed solution involves the second order differential operator without Fredholm property.
\end{abstract}

\section{Introduction}

Let us consider the problem

$$
-\Delta u+V(x) u-a u=f,
$$

where $u \in E=H^{2}\left(\mathbf{R}^{d}\right)$ and $f \in F=L^{2}\left(\mathbf{R}^{d}\right), d \in \mathbf{N}, a$ is a constant and the scalar potential function $V(x)$ either vanishes or converges to 0 at infinity. In the case of $a \geq 0$, the essential spectrum of the operator $A: E \rightarrow F$ correspondent to the lefthand side of equation (1.1) contains the origin. Consequently, this operator fails to satisfy the Fredholm property. Its image is not closed, for $d>1$ the dimensions of its kernel and the codimension of its image are not finite. The present work is devoted to the studies of certain properties of such operators. Let us note that elliptic problems involving operators without Fredholm property were studied extensively in recent years. Solvability conditions in weighted Sobolev and Hölder spaces were obtained in $[2,3,4,5,6]$. The Schrödinger type operators without Fredholm property were treated via the methods of the spectral and the scattering theory in $[12,13,15,16,17]$. The Laplacian operator with drift from the point of view of non Fredholm operators was studied in [20] and linearized Cahn-Hilliard equations in [18] and [21]. Nonlinear non Fredholm elliptic problems were treated in [19] and [22]. Potential applications to the theory of reaction-diffusion equations were explored in $[9,10]$. Non Fredholm operators arise also when studying wave systems with an infinite number of localized traveling waves (see [1]).

One of the important questions about problems with non-Fredholm operators concerns their solvability. We will consider the nonlinear heat equation

$$
\frac{\partial u}{\partial t}=\Delta u+\varepsilon g(u)+f(x), \quad x \in \mathbf{R}^{5}
$$

doi:10.5186/aasfm.2015.4013

2010 Mathematics Subject Classification: Primary 35J05, 35P30, 47 F05.

Key words: Nonlinear heat equations, non Fredholm operators, Sobolev spaces. 
with the parameter $\varepsilon \geq 0$. Seeking the stationary solutions of problem (1.2) yields the nonlinear Poisson equation

$$
-\Delta u=f(x)+\varepsilon g(u) .
$$

Let us make the following technical assumption about the source term of problem (1.3).

Assumption 1. Let $f(x): \mathbf{R}^{5} \rightarrow \mathbf{R}$ be nontrivial, $f(x) \in L^{1}\left(\mathbf{R}^{5}\right)$ and $\nabla f(x) \in$ $L^{2}\left(\mathbf{R}^{5}\right)$.

Note that by means of the Sobolev inequality (see e.g. p. 183 of [11]) under the assumption above we have

$$
f(x) \in L^{2}\left(\mathbf{R}^{5}\right) .
$$

We will be using the Sobolev space

$$
H^{3}\left(\mathbf{R}^{5}\right)=\left\{u(x): \mathbf{R}^{5} \rightarrow \mathbf{C} \mid u(x) \in L^{2}\left(\mathbf{R}^{5}\right),(-\Delta)^{\frac{3}{2}} u \in L^{2}\left(\mathbf{R}^{5}\right)\right\}
$$

equipped with the norm

$$
\|u\|_{H^{3}\left(\mathbf{R}^{5}\right)}^{2}=\|u\|_{L^{2}\left(\mathbf{R}^{5}\right)}^{2}+\left\|(-\Delta)^{\frac{3}{2}} u\right\|_{L^{2}\left(\mathbf{R}^{5}\right)}^{2} .
$$

The operator $(-\Delta)^{\frac{3}{2}}$ is defined via the spectral calculus. By means of the Sobolev embedding we have

$$
\|u\|_{L^{\infty}\left(\mathbf{R}^{5}\right)} \leq c_{e}\|u\|_{H^{3}\left(\mathbf{R}^{5}\right)},
$$

where $c_{e}>0$ is the constant of the embedding. The hat symbol will stand for the standard Fourier transform, such that

$$
\widehat{u}(p)=\frac{1}{(2 \pi)^{\frac{5}{2}}} \int_{\mathbf{R}^{5}} u(x) e^{-i p x} d x .
$$

This enables us to express the Sobolev norm as

$$
\|u\|_{H^{3}\left(\mathbf{R}^{5}\right)}^{2}=\int_{\mathbf{R}^{5}}\left(1+|p|^{6}\right)|\widehat{u}(p)|^{2} d p .
$$

When the parameter $\varepsilon$ vanishes, we arrive at the standard Poisson equation

$$
-\Delta u=f(x) \text {. }
$$

Under Assumption 1 by means of Lemma 7 of [22] problem (1.8) admits a unique solution $u_{0}(x) \in H^{2}\left(\mathbf{R}^{5}\right)$ and no orthogonality relations are required. As discussed in Lemmas 5 and 6 of [22], in dimensions $d<5$ we need certain orthogonality conditions for the solvability of equation (1.8) in $H^{2}\left(\mathbf{R}^{d}\right)$. We do not discuss the problem in dimensions $d>5$ to avoid extra technicalities since the argument will rely on similar ideas (see Lemma 7 of [22]). Due to our Assumption 1

$$
\nabla(-\Delta u)=\nabla f(x) \in L^{2}\left(\mathbf{R}^{5}\right) .
$$

Therefore, for the unique solution of the linear Poisson equation (1.8) we have $u_{0}(x) \in$ $H^{3}\left(\mathbf{R}^{5}\right)$. By seeking the resulting solution of the nonlinear Poisson equation (1.3) as

$$
u(x)=u_{0}(x)+u_{p}(x)
$$

we clearly arrive at the perturbative equation

$$
-\Delta u_{p}=\varepsilon g\left(u_{0}+u_{p}\right) \text {. }
$$


Let us introduce a closed ball in our Sobolev space

$$
B_{\rho}:=\left\{u(x) \in H^{3}\left(\mathbf{R}^{5}\right) \mid\|u\|_{H^{3}\left(\mathbf{R}^{5}\right)} \leq \rho\right\}, \quad 0<\rho \leq 1 .
$$

We will seek the solution of (1.10) as the fixed point of the auxiliary nonlinear problem

$$
-\Delta u=\varepsilon g\left(u_{0}+v\right) \text {. }
$$

in the ball (1.11). Note that the left side of (1.12) involves the operator $-\Delta$ : $H^{2}\left(\mathbf{R}^{5}\right) \rightarrow L^{2}\left(\mathbf{R}^{5}\right)$, which has no Fredholm property, since its essential spectrum fills the nonnegative semi-axis $[0,+\infty)$ and therefore, a bounded inverse of this operator does not exist. The similar situation arised in [19] and [22] but as distinct from the present work, the problems treated there were nonlocal. The fixed point technique was used in [14] to estimate the perturbation to the standing solitary wave of the Nonlinear Schrödinger (NLS) equation when either the external potential or the nonlinear term in the NLS were perturbed but the Schrödinger type operator involved in such nonlinear problem possessed the Fredholm property (see Assumption 1 of [14], also [7]). Let us define the interval on the real line

$$
I:=\left[-c_{e}\left\|u_{0}\right\|_{H^{3}\left(\mathbf{R}^{5}\right)}-c_{e}, \quad c_{e}\left\|u_{0}\right\|_{H^{3}\left(\mathbf{R}^{5}\right)}+c_{e}\right] .
$$

We make the following assumption about the nonlinear part of problem (1.3).

Assumption 2. Let $g(s): \mathbf{R} \rightarrow \mathbf{R}$, such that $g(0)=0$ and $g^{\prime}(0)=0$. We also assume that $g(s) \in C_{2}(\mathbf{R})$, such that

$$
a_{2}:=\sup _{s \in I}\left|g^{\prime \prime}(s)\right|>0 .
$$

Note that $a_{1}:=\sup _{s \in I}\left|g^{\prime}(s)\right|>0$ as well, otherwise the function $g(s)$ will be constant on the interval $I$ and $a_{2}$ will vanish. For instance, $g(s)=s^{2}$ clearly satisfies the assumption above. Our main statement is as follows.

Theorem 3. Let Assumptions 1 and 2 hold. Then equation (1.12) defines the map $T_{g}: B_{\rho} \rightarrow B_{\rho}$, which is a strict contraction for all $0<\varepsilon<\varepsilon *$ for a certain $\varepsilon *>0$. The unique fixed point $u_{p}(x)$ of the map $T_{g}$ is the only solution of problem (1.10) in $B_{\rho}$.

Note that the resulting solution of problem (1.3) given by (1.9) will be nontrivial since the source term $f(x)$ is nontrivial and $g(0)$ vanishes according to our assumptions. We will make use of the following elementary technical lemma.

Lemma 4. Consider the function $\varphi(R):=\alpha R+\frac{\beta}{R^{4}}$ on the positive semi-axis $(0,+\infty)$ with the constants $\alpha, \beta>0$. It attains the minimal value at $R^{*}=\left(\frac{4 \beta}{\alpha}\right)^{\frac{1}{5}}$, which is given by $\varphi\left(R^{*}\right)=\frac{5}{4^{\frac{4}{5}}} \alpha^{\frac{4}{5}} \beta^{\frac{1}{5}}$.

Let us proceed to the proof of our main result.

\section{The existence of the perturbed solution}

Proof of Theorem 3. Let us choose arbitrarily $v(x) \in B_{\rho}$ and denote the right side of equation (1.12) as $G(x):=g\left(u_{0}+v\right)$. By applying the standard Fourier transform (1.6) to both sides of problem (1.12), we arrive at

$$
\widehat{u}(p)=\varepsilon \frac{\widehat{G}(p)}{p^{2}},
$$


such that for the norm we have

$$
\|u\|_{L^{2}\left(\mathbf{R}^{5}\right)}^{2}=\varepsilon^{2} \int_{\mathbf{R}^{5}} \frac{|\widehat{G}(p)|^{2}}{|p|^{4}} d p .
$$

Clearly,

$$
\|\widehat{G}(p)\|_{L^{\infty}\left(\mathbf{R}^{5}\right)} \leq \frac{1}{(2 \pi)^{\frac{5}{2}}}\|G(x)\|_{L^{1}\left(\mathbf{R}^{5}\right)} .
$$

Let us estimate the right side of (2.1) using (2.2) with $R>0$ as

$$
\begin{aligned}
& \varepsilon^{2} \int_{|p| \leq R} \frac{|\widehat{G}(p)|^{2}}{|p|^{4}} d p+\varepsilon^{2} \int_{|p|>R} \frac{|\widehat{G}(p)|^{2}}{|p|^{4}} d p \\
& \leq \varepsilon^{2} \frac{1}{(2 \pi)^{5}}\|G(x)\|_{L^{1}\left(\mathbf{R}^{5}\right)}^{2}\left|S_{5}\right| R+\varepsilon^{2} \frac{1}{R^{4}}\|G(x)\|_{L^{2}\left(\mathbf{R}^{5}\right)}^{2} .
\end{aligned}
$$

Here and below $S_{5}$ stands for the unit sphere in the space of five dimensions centered at the origin and $\left|S_{5}\right|$ for its Lebesgue measure (see e.g. p. 6 of [11]). Since $v(x) \in B_{\rho}$, we have

$$
\left\|u_{0}+v\right\|_{L^{2}\left(\mathbf{R}^{5}\right)} \leq\left\|u_{0}\right\|_{H^{3}\left(\mathbf{R}^{5}\right)}+1 \text {. }
$$

Also, the Sobolev embedding (1.5) yields

$$
\left|u_{0}+v\right| \leq c_{e}\left\|u_{0}\right\|_{H^{3}\left(\mathbf{R}^{5}\right)}+c_{e} .
$$

Using the representation $G(x)=\int_{0}^{u_{0}+v} g^{\prime}(s) d s$, with the interval $I$ given by (1.13), we easily obtain

$$
|G(x)| \leq \sup _{s \in I}\left|g^{\prime}(s)\right|\left|u_{0}+v\right|=a_{1}\left|u_{0}+v\right|,
$$

such that

$$
\|G(x)\|_{L^{2}\left(\mathbf{R}^{5}\right)} \leq a_{1}\left\|u_{0}+v\right\|_{L^{2}\left(\mathbf{R}^{5}\right)} \leq a_{1}\left(\left\|u_{0}\right\|_{H^{3}\left(\mathbf{R}^{5}\right)}+1\right) .
$$

Similarly, $G(x)=\int_{0}^{u_{0}+v} d s\left[\int_{0}^{s} g^{\prime \prime}(t) d t\right]$. Therefore, we estimate

$$
\begin{aligned}
|G(x)| & \leq \frac{1}{2} \sup _{t \in I}\left|g^{\prime \prime}(t) \| u_{0}+v\right|^{2}=\frac{a_{2}}{2}\left|u_{0}+v\right|^{2}, \\
\|G(x)\|_{L^{1}\left(\mathbf{R}^{5}\right)} & \leq \frac{a_{2}}{2}\left\|u_{0}+v\right\|_{L^{2}\left(\mathbf{R}^{5}\right)}^{2} \leq \frac{a_{2}}{2}\left(\left\|u_{0}\right\|_{H^{3}\left(\mathbf{R}^{5}\right)}+1\right)^{2} .
\end{aligned}
$$

Thus we arrive at the upper bound for the right side of (2.3) as

$$
\frac{\varepsilon^{2}}{(2 \pi)^{5}}\left|S_{5}\right| \frac{a_{2}^{2}}{4}\left(\left\|u_{0}\right\|_{H^{3}\left(\mathbf{R}^{5}\right)}+1\right)^{4} R+\varepsilon^{2} a_{1}^{2}\left(\left\|u_{0}\right\|_{H^{3}\left(\mathbf{R}^{5}\right)}+1\right)^{2} \frac{1}{R^{4}}
$$

with $R \in(0,+\infty)$. By means of Lemma 4 we obtain the minimal value of the expression above. Hence

$$
\|u\|_{L^{2}\left(\mathbf{R}^{5}\right)}^{2} \leq \varepsilon^{2} \frac{\left|S_{5}\right|^{\frac{4}{5}}}{(2 \pi)^{4}} a_{2}^{\frac{8}{5}}\left(\left\|u_{0}\right\|_{H^{3}\left(\mathbf{R}^{5}\right)}+1\right)^{3 \frac{3}{5}} a_{1}^{\frac{2}{5}} \frac{5}{4^{\frac{8}{5}}} .
$$

Clearly, (1.12) implies that

$$
\nabla(-\Delta u)=\varepsilon g^{\prime}\left(u_{0}+v\right)\left(\nabla u_{0}+\nabla v\right) .
$$

We will make use of the identity

$$
g^{\prime}\left(u_{0}+v\right)=\int_{0}^{u_{0}+v} g^{\prime \prime}(s) d s
$$


along with the Sobolev embedding (1.5), such that

$$
\left|g^{\prime}\left(u_{0}+v\right)\right| \leq \sup _{s \in I}\left|g^{\prime \prime}(s)\right|\left|u_{0}+v\right| \leq a_{2} c_{e}\left(\left\|u_{0}\right\|_{H^{3}\left(\mathbf{R}^{5}\right)}+1\right)
$$

and

$$
|\nabla(-\Delta u)| \leq \varepsilon a_{2} c_{e}\left(\left\|u_{0}\right\|_{H^{3}\left(\mathbf{R}^{5}\right)}+1\right)\left|\nabla u_{0}+\nabla v\right| .
$$

Using the inequality, which can be trivially derived via the standard Fourier transform, namely

$$
\|\nabla u\|_{L^{2}\left(\mathbf{R}^{5}\right)} \leq\|u\|_{H^{3}\left(\mathbf{R}^{5}\right)},
$$

we easily arrive at

$$
\left\|(-\Delta)^{\frac{3}{2}} u\right\|_{L^{2}\left(\mathbf{R}^{5}\right)}^{2} \leq \varepsilon^{2} a_{2}^{2} c_{e}^{2}\left(\left\|u_{0}\right\|_{H^{3}\left(\mathbf{R}^{5}\right)}+1\right)^{4} .
$$

By virtue of the definition of the norm (1.4) along with estimates (2.4) and (2.6) we derive

$$
\|u\|_{H^{3}\left(\mathbf{R}^{5}\right)} \leq \varepsilon\left(\left\|u_{0}\right\|_{H^{3}\left(\mathbf{R}^{5}\right)}+1\right)^{2} a_{2}^{\frac{4}{5}} \sqrt{\frac{\left|S_{5}\right|^{\frac{4}{5}}}{(2 \pi)^{4}} a_{1}^{\frac{2}{5}} \frac{5}{4^{\frac{8}{5}}}+a_{2}^{\frac{2}{5}} c_{e}^{2}} \leq \rho
$$

for all positive values of the parameter $\varepsilon$ small enough, such that $u(x) \in B_{\rho}$ as well. Suppose for some $v(x) \in B_{\rho}$ there are two solutions $u_{1,2}(x) \in B_{\rho}$ of problem (1.12). Then their difference $u(x):=u_{1}(x)-u_{2}(x) \in L^{2}\left(\mathbf{R}^{5}\right)$ satisfies the Laplace equation. Since there are no nontrivial square integrable harmonic functions, $u(x)=0$ a.e. in $\mathbf{R}^{5}$. Therefore, equation (1.12) defines a map $T_{g}: B_{\rho} \rightarrow B_{\rho}$ when $\varepsilon>0$ is sufficiently small.

Let us show that this map is a strict contraction. We choose arbitrarily $v_{1,2}(x) \in$ $B_{\rho}$, such that by virtue of the argument above $u_{1,2}=T_{g} v_{1,2} \in B_{\rho}$ as well. Explicitly, via (1.12) we have

$$
-\Delta u_{1}=\varepsilon g\left(u_{0}+v_{1}\right), \quad-\Delta u_{2}=\varepsilon g\left(u_{0}+v_{2}\right) .
$$

Let us introduce

$$
G_{1}(x):=g\left(u_{0}+v_{1}\right), \quad G_{2}(x):=g\left(u_{0}+v_{2}\right) .
$$

Then by applying the standard Fourier transform (1.6) to both sides of each of the equations (2.7), we obtain

$$
\widehat{u_{1}}(p)=\varepsilon \frac{\widehat{G_{1}}(p)}{p^{2}}, \quad \widehat{u_{2}}(p)=\varepsilon \frac{\widehat{G_{2}}(p)}{p^{2}} .
$$

Therefore, we express the norm

$$
\left\|u_{1}-u_{2}\right\|_{L^{2}\left(\mathbf{R}^{5}\right)}^{2}=\varepsilon^{2} \int_{\mathbf{R}^{5}} \frac{\left|\widehat{G_{1}}(p)-\widehat{G_{2}}(p)\right|^{2}}{|p|^{4}} d p,
$$

which can be estimated via (2.2) as

$$
\begin{aligned}
& \varepsilon^{2} \int_{|p| \leq R} \frac{\left|\widehat{G_{1}}(p)-\widehat{G_{2}}(p)\right|^{2}}{|p|^{4}} d p+\varepsilon^{2} \int_{|p|>R} \frac{\left|\widehat{G_{1}}(p)-\widehat{G_{2}}(p)\right|^{2}}{|p|^{4}} d p \\
& \leq \frac{\varepsilon^{2}}{(2 \pi)^{5}}\left\|G_{1}(x)-G_{2}(x)\right\|_{L^{1}\left(\mathbf{R}^{5}\right)}^{2}\left|S_{5}\right| R+\frac{\varepsilon^{2}}{R^{4}}\left\|G_{1}(x)-G_{2}(x)\right\|_{L^{2}\left(\mathbf{R}^{5}\right)}^{2}
\end{aligned}
$$


with $R \in(0,+\infty)$. Let us make use of the representation

$$
G_{1}(x)-G_{2}(x)=\int_{u_{0}+v_{2}}^{u_{0}+v_{1}} g^{\prime}(s) d s
$$

such that

$$
\left|G_{1}(x)-G_{2}(x)\right| \leq \sup _{s \in I}\left|g^{\prime}(s)\right|\left|v_{1}-v_{2}\right|=a_{1}\left|v_{1}-v_{2}\right|
$$

and therefore

$$
\left\|G_{1}(x)-G_{2}(x)\right\|_{L^{2}\left(\mathbf{R}^{5}\right)} \leq a_{1}\left\|v_{1}-v_{2}\right\|_{L^{2}\left(\mathbf{R}^{5}\right)} \leq a_{1}\left\|v_{1}-v_{2}\right\|_{H^{3}\left(\mathbf{R}^{5}\right)} .
$$

We can also express

$$
G_{1}(x)-G_{2}(x)=\int_{u_{0}+v_{2}}^{u_{0}+v_{1}} d s\left[\int_{0}^{s} g^{\prime \prime}(t) d t\right] .
$$

This enables us to estimate $G_{1}(x)-G_{2}(x)$ in the absolute value from above by

$$
\frac{1}{2} \sup _{t \in I}\left|g^{\prime \prime}(t)\right|\left|\left(v_{1}-v_{2}\right)\left(2 u_{0}+v_{1}+v_{2}\right)\right|=\frac{a_{2}}{2}\left|\left(v_{1}-v_{2}\right)\left(2 u_{0}+v_{1}+v_{2}\right)\right| .
$$

Via the Schwarz inequality we derive the upper bound for the norm $\| G_{1}(x)-$ $G_{2}(x) \|_{L^{1}\left(\mathbf{R}^{5}\right)}$ as

$$
\frac{a_{2}}{2}\left\|v_{1}-v_{2}\right\|_{L^{2}\left(\mathbf{R}^{5}\right)}\left\|2 u_{0}+v_{1}+v_{2}\right\|_{L^{2}\left(\mathbf{R}^{5}\right)} \leq a_{2}\left\|v_{1}-v_{2}\right\|_{H^{3}\left(\mathbf{R}^{5}\right)}\left(\left\|u_{0}\right\|_{H^{3}\left(\mathbf{R}^{5}\right)}+1\right) .
$$

Thus we arrive at

$$
\left\|u_{1}(x)-u_{2}(x)\right\|_{L^{2}\left(\mathbf{R}^{5}\right)}^{2} \leq \varepsilon^{2}\left\|v_{1}-v_{2}\right\|_{H^{3}\left(\mathbf{R}^{5}\right)}^{2}\left\{\frac{a_{2}^{2}}{(2 \pi)^{5}}\left(\left\|u_{0}\right\|_{H^{3}\left(\mathbf{R}^{5}\right)}+1\right)^{2}\left|S_{5}\right| R+\frac{a_{1}^{2}}{R^{4}}\right\} .
$$

Lemma 4 enables us to minimize the right side of the inequality above over $R>0$, such that we obtain

$$
\left\|u_{1}(x)-u_{2}(x)\right\|_{L^{2}\left(\mathbf{R}^{5}\right)}^{2} \leq \varepsilon^{2}\left\|v_{1}-v_{2}\right\|_{H^{3}\left(\mathbf{R}^{5}\right)}^{2} \frac{5}{4^{\frac{4}{5}}} \frac{a_{2}^{\frac{8}{5}}}{(2 \pi)^{4}}\left(\left\|u_{0}\right\|_{H^{3}\left(\mathbf{R}^{5}\right)}+1\right)^{2}\left|S_{5}\right|^{\frac{4}{5}} a_{1}^{\frac{2}{5}} .
$$

Using (2.7) we express $\nabla(-\Delta)\left(u_{1}-u_{2}\right)$ as

$$
\begin{aligned}
& \varepsilon\left[g^{\prime}\left(u_{0}+v_{1}\right)\left(\nabla u_{0}+\nabla v_{1}\right)-g^{\prime}\left(u_{0}+v_{2}\right)\left(\nabla u_{0}+\nabla v_{2}\right)\right] \\
& =\varepsilon\left[\left(\nabla u_{0}+\nabla v_{1}\right) \int_{u_{0}+v_{2}}^{u_{0}+v_{1}} g^{\prime \prime}(s) d s+\left(\nabla v_{1}-\nabla v_{2}\right) \int_{0}^{u_{0}+v_{2}} g^{\prime \prime}(s) d s\right] .
\end{aligned}
$$

This yields the upper bound for $\left|\nabla(-\Delta)\left(u_{1}-u_{2}\right)\right|$ as

$$
\varepsilon \sup _{s \in I}\left|g^{\prime \prime}(s)\right|\left|v_{1}-v_{2}\right|\left|\nabla u_{0}+\nabla v_{1}\right|+\varepsilon \sup _{s \in I}\left|g^{\prime \prime}(s)\right|\left|u_{0}+v_{2}\right|\left|\nabla v_{1}-\nabla v_{2}\right|,
$$

which can be easily estimated from above by virtue of the Sobolev embedding (1.5) by

$$
\varepsilon a_{2} c_{e}\left\|v_{1}-v_{2}\right\|_{H^{3}\left(\mathbf{R}^{5}\right)}\left|\nabla u_{0}+\nabla v_{1}\right|+\varepsilon a_{2} c_{e}\left\|u_{0}+v_{2}\right\|_{H^{3}\left(\mathbf{R}^{5}\right)}\left|\nabla v_{1}-\nabla v_{2}\right|,
$$
such that

$$
\begin{aligned}
\left\|\nabla(-\Delta)\left(u_{1}-u_{2}\right)\right\|_{L^{2}\left(\mathbf{R}^{5}\right)} \leq & \varepsilon a_{2} c_{e}\left\|v_{1}-v_{2}\right\|_{H^{3}\left(\mathbf{R}^{5}\right)}\left\|\nabla u_{0}+\nabla v_{1}\right\|_{L^{2}\left(\mathbf{R}^{5}\right)} \\
& +\varepsilon a_{2} c_{e}\left(\left\|u_{0}\right\|_{H^{3}\left(\mathbf{R}^{5}\right)}+1\right)\left\|\nabla v_{1}-\nabla v_{2}\right\|_{L^{2}\left(\mathbf{R}^{5}\right)}
\end{aligned}
$$

By virtue of (2.5) using that $v_{1} \in B_{\rho}$ we arrive at

$$
\left\|\nabla(-\Delta)\left(u_{1}-u_{2}\right)\right\|_{L^{2}\left(\mathbf{R}^{5}\right)}^{2} \leq 4 \varepsilon^{2} a_{2}^{2} c_{e}^{2}\left(\left\|u_{0}\right\|_{H^{3}\left(\mathbf{R}^{5}\right)}+1\right)^{2}\left\|v_{1}-v_{2}\right\|_{H^{3}\left(\mathbf{R}^{5}\right)}^{2} .
$$


Inequalities (2.8) and (2.9) imply that

$$
\left\|u_{1}-u_{2}\right\|_{H^{3}\left(\mathbf{R}^{5}\right)} \leq \varepsilon\left(\left\|u_{0}\right\|_{H^{3}\left(\mathbf{R}^{5}\right)}+1\right) a_{2}^{\frac{4}{5}}\left[\frac{5}{4^{\frac{4}{5}}} \frac{a_{1}^{\frac{2}{5}}}{(2 \pi)^{4}}\left|S_{5}\right|^{\frac{4}{5}}+4 a_{2}^{\frac{2}{5}} c_{e}^{2}\right]^{\frac{1}{2}}\left\|v_{1}-v_{2}\right\|_{H^{3}\left(\mathbf{R}^{5}\right)} .
$$

Therefore, the map $T_{g}: B_{\rho} \rightarrow B_{\rho}$ defined by equation (1.12) is a strict contraction for all values of $\varepsilon>0$ sufficiently small. Its unique fixed point $u_{p}(x)$ is the only solution of problem (1.10) in $B_{\rho}$, such that the resulting $u(x) \in H^{3}\left(\mathbf{R}^{5}\right)$ given by (1.9) is the stationary solution of our nonlinear heat equation (1.2).

Acknowledgements. Valuable discussions with D. Pelinovsky are gratefully acknowledged.

\section{References}

[1] Alfimov, G. L., E. V. Medvedeva, and D. E. Pelinovsky: Wave systems with an infinite number of localized traveling waves. - Phys. Rev. Lett. 112, 2014, 054103, 1-5.

[2] Amrouche, C., and F. Bonzom: Mixed exterior Laplace's problem. - J. Math. Anal. Appl. $338,2008,124-140$.

[3] Amrouche, C., V. Girault, and J. Giroire: Dirichlet and Neumann exterior problems for the $n$-dimensional Laplace operator. An approach in weighted Sobolev spaces. - J. Math. Pures Appl. 76, 1997, 55-81.

[4] Benkirane, N.: Propriété d'indice en théorie Holderienne pour des opérateurs elliptiques dans $\mathbf{R}^{n}$. - CRAS Série I 307, 1988, 577-580.

[5] Bolley, P., and T. L. Pham: Propriété d'indice en théorie Holderienne pour des opérateurs différentiels elliptiques dans $\mathbf{R}^{n}$. - J. Math. Pures Appl. 72, 1993, 105-119.

[6] Bolley, P., and T. L. Pham: Propriété d'indice en théorie Hölderienne pour le problème extérieur de Dirichlet. - Comm. Partial Differential Equations 26:1-2, 2001, 315-334.

[7] Cuccagna, S., D. Pelinovsky, and V. Vougalter: Spectra of positive and negative energies in the linearized NLS problem. - Comm. Pure Appl. Math. 58:1, 2005, 1-29.

[8] Cycon, H. L., R. G. Froese, W. Kirsch, and B. Simon: Schrödinger operators with application to quantum mechanics and global geometry. - Springer-Verlag, Berlin, 1987.

[9] Ducrot, A., M. Marion, and V. Volpert: Systemes de réaction-diffusion sans propriété de Fredholm. - CRAS 340, 2005, 659-664.

[10] Ducrot, A., M. Marion, and V. Volpert: Reaction-diffusion problems with non Fredholm operators. - Adv. Differential Equations 13:11-12, 2008, 1151-1192.

[11] Lieb, E., and M. Loss: Analysis. - Grad. Stud. Math. 14, Amer. Math. Soc., Providence, 1997.

[12] Volpert, V.: Elliptic partial differential equations. Volume 1. Fredholm theory of elliptic problems in unbounded domains. - Birkhauser, 2011.

[13] Volpert, V., B. Kazmierczak, M. Massot, and Z. Peradzynski: Solvability conditions for elliptic problems with non-Fredholm operators. - Appl. Math. 29:2, 2002, 219-238.

[14] Vougalter, V.: On threshold eigenvalues and resonances for the linearized NLS equation. Math. Model. Nat. Phenom. 5:4, 2010, 448-469.

[15] Vougalter, V., and V. Volpert: On the solvability conditions for some non Fredholm operators. - Int. J. Pure Appl. Math. 60:2, 2010, 169-191.

[16] Vougalter, V., and V. Volpert: Solvability relations for some non Fredholm operators. Int. Electron. J. Pure Appl. Math. 2:1, 2010, 75-83. 
[17] Vougalter, V., and V. Volpert: Solvability conditions for some non-Fredholm operators. - Proc. Edinb. Math. Soc. (2) 54:1, 2011, 249-271

[18] Volpert, V., and V. Vougalter: On the solvability conditions for a linearized Cahn-Hilliard equation. - Rend. Istit. Mat. Univ. Trieste 43, 2011, 1-9.

[19] Vougalter, V., and V. Volpert: On the existence of stationary solutions for some nonFredholm integro-differential equations. Doc. Math. 16, 2011, 561-580.

[20] Vougalter, V., and V. Volpert: On the solvability conditions for the diffusion equation with convection terms. - Commun. Pure Appl. Anal. 11:1, 2012, 365-373.

[21] Vougalter, V., and V. Volpert: Solvability conditions for a linearized Cahn-Hilliard equation of sixth order. - Math. Model. Nat. Phenom. 7:2, 2012, 146-154.

[22] Vougalter, V., and V. Volpert: Solvability conditions for some linear and nonlinear nonFredholm elliptic problems. - Anal. Math. Phys. 2:4, 2012, 473-496.

Received 11 July $2014 \bullet$ Accepted 22 August 2014 